\title{
Alfvén wings at Earth's magnetosphere under strong interplanetary magnetic fields
}

\author{
A. J. Ridley \\ University of Michigan, Ann Arbor, MI, USA
}

Received: 9 August 2006 - Revised: 4 December 2006 - Accepted: 10 January 2007 - Published: 8 March 2007

\begin{abstract}
A number of recent studies have shown that the upstream Mach number may play a significant role in the energy transfer between the solar wind and the magnetosphere. Magnetohydrodynamic (MHD) simulation results of the magnetosphere-ionosphere system are presented that show the transition from nominal solar wind and interplanetary magnetic field driving to extremely strong driving. One of the predominant features of the magnetosphere that becomes apparent during low Mach number conditions is the formation of Alfvén wings above and below the magnetosphere. Alfvén wing are cavities of low flow, and have been observed at Io and Ganymede, both of which reside in regions of sub-Alfvénic flow. It is shown that Alfvén wings exist even during nominal Mach number time periods - the wings fold over to form what has been classically viewed as the magnetotail. The regions of low flow within the Alfvén wing limit the electric field applied across the ionosphere, hence causing the ionospheric cross polar cap potential to be dependent upon the Mach number, and in turn, causing the saturation of the potential.
\end{abstract}

Keywords. Ionosphere (Electric fields and currents) - Magnetospheric physics (Polar cap phenomena; Solar windmagnetosphere interactions)

\section{Introduction}

In the past few years, only a hand-full of extremely strong solar wind driven events have occurred. For example, during the super storm of 14 June 2000, the interplanetary magnetic field (IMF) $B_{z}$ dipped down to $-60 \mathrm{nT}$, while the density rose to almost $100 \mathrm{~cm}^{-3}$ during the sheath of the coronal mass ejection (CME). Inside the CME proper, the magnetic field became very strong, while the density became very low, typ-

Correspondence to: A. J. Ridley

(ridley@umich.edu) ical of CMEs. During this event, the solar wind became subAlfvénic (i.e. a Mach number of less than one). The Alfvén Mach number is one of the strong controlling factors of the behavior of a plasma embedded in a magnetic field. In the typical solar wind, the Alfvén Mach number is 8 or above. In the middle of a CME, the Mach number can become quite reduced, reaching values of 2 or less. It is therefore reasonable to investigate how the magnetosphere reacts under these types of flow conditions, and to understand if some of the features observed during major storms are due to the Mach number, instead of internal mechanisms.

Other solar system bodies exist in sub-Alfvénic flow regimes most of the time, and have been examined by theory, modelling and utilizing satellite data. For example, both Io (Neubauer, 1980; Linker et al., 1998) and Ganymede (Ip and Kopp, 2002; Kopp and Ip, 2002) exist in Jupiter's low density, high field strength magnetosphere. Each exhibits the formation of "Alfvén wings" or "Alfvén tubes", as described by Drell et al. (1965) and later by Neubauer (1980). Alfvén wings are a consequence of the interaction between the flow velocity and an Alfvén wave. As a magnetic field encounters an obstacle and starts to bend, Alfvén waves are launched along the field-lines away from that point. These Alfvén waves propagate along the magnetic field line with the speed $V_{A}=B / \sqrt{\mu_{0} \rho}$, where $B$ is the magnetic field strength, and $\rho$ is the plasma mass density. In addition, the plasma still advects the magnetic field with a given velocity $V$. The Alfvén wave, therefore, travels with an angle $\theta=\operatorname{atan}\left(M_{A}^{-1}\right)$, where $M_{A}$ is the Alfvén Mach number, defined by $M_{A}=V / V_{A}$ (Neubauer, 1980). The flow diverts around the obstacle and forms two tubes (above and below) the object in which the flow characteristics of the plasma are altered significantly from the surrounding medium. This cavity is the Alfvén wing. At very low Mach numbers, these wings are at a high angle, so they are easy to identify in satellite data (e.g. Kivelson et al., 1997). At Jupiter, this creates an interaction between the currents formed by the Alfvén wings at each of

Published by Copernicus GmbH on behalf of the European Geosciences Union. 

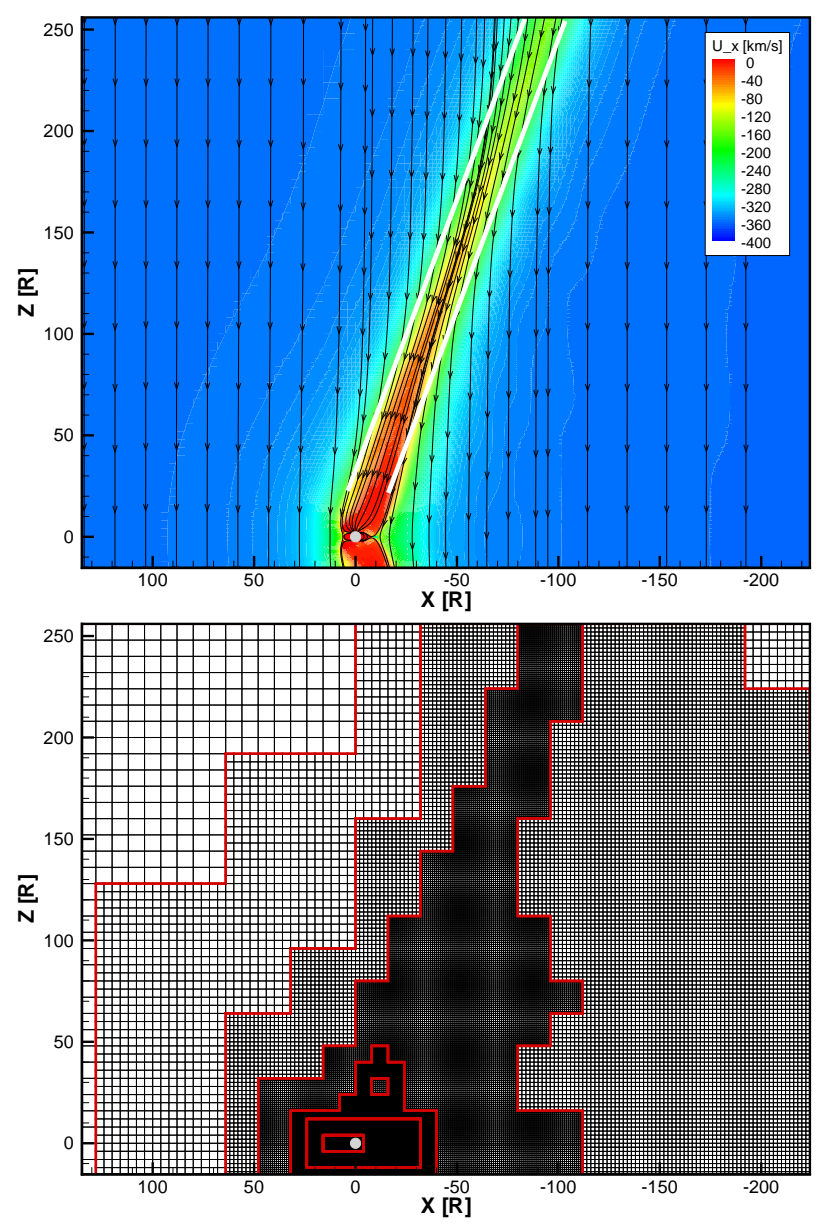

Fig. 1. BATSRUS simulation of sub-Alfvénic flow at the Earth $\left(B_{z}=-100 \mathrm{nT}\right)$. (Top) The block adaptive mesh used in the simulation with red lines indicating boundaries between different resolutions. The grid is easily mapped with high resolution along the Alfvén wing. (Bottom) Magnetic field lines in black over color contours of the flow velocity for the same simulation. The slowing of the flow in the Alfvén wing is clearly seen.

the moons and Jupiter's ionosphere (e.g. Crary and Bagenal, 1997). Interestingly, Io has no magnetic field, so the formation of Alfvén wings is not dependent upon being a magnetized body, and Ganymede has no real ionosphere, so the formation of the Alfvén wings is not contingent upon having an ionospheric conductance, although some characteristics of the wing may be dependent upon the ionospheric conductivity.

Figure 1 shows an example of an Alfvén wing and the grid structure in an Earth magnetospheric simulation where the interplanetary magnetic field (IMF) $B_{z}$ is $-100 \mathrm{nT}$. The contour shows the flow velocity in the sunward direction. The blue region is near the solar wind flow velocity, while the red region is the Alfvén wing (further indicated by the white lines). In this region, the flow velocity is significantly re- duced. Magnetic field lines are shown, indicating that the magnetic field bends sharply as it enters the Alfvén wing, and bends back as it leaves the wing. These are the outstanding characteristics of the Alfvén wing.

For Mach numbers greater than 1, the Alfvén wing angle would be shallower. At Earth, the nominal Mach number is around 8 , which would produce an extremely shallow Alfvén wing. Indeed, researchers may not recognize it as being an Alfvén wing at all. The upper and lower wings would essentially touch, forming the magnetotail, where the current sheet is the separation between the two wings that have been folded over. The purpose of this study is to examine the transition of the magnetospheric domain from being driven by a nominal Mach number solar wind (i.e., around eight) to being driven by a low Mach number solar wind (i.e., less than one). The currents in the vicinity of the magnetopause, the magnetospheric electric field, and the ionospheric cross polar cap potential are examined.

\section{Technique}

The University of Michigan's magnetohydrodynamic code was described by Powell et al. (1999), while the magnetosphere - ionosphere coupling within the code was described by Ridley et al. (2004). The MHD code has a block based structure, such that a wide range of scale sizes can be simulated within the same domain. In the simulations presented here, the inner boundary of the simulation is at $2.5 R_{E}$ (Earth radii), which is resolved with cells $1 / 8 R_{E}$ in size. The reconnection site on the dayside is also resolved with $1 / 8 R_{E}$ grid cells. The Alfvén wings, described below, are resolved with $1 / 4 R_{E}$ grid cells in the near Earth region and grid cells $1 R_{E}$ in size to the top of the simulation domain. Each simulation has approximately 10 million cells.

Figure 1 shows the grid structure of a simulation of an Alfvén wing. Grid-based bias in the solution was removed by utilizing the adaptive mesh refinement within the MHD code. This allowed the solution to be resolved with higher resolution without presupposing the location of the wing structure (or even the presupposition of the existence of the structure). An initial, moderately resolved solution was allowed to converge for many iterations, then $10 \%$ more cells were added to the simulation where the currents and gradients in velocity were largest. The solution was then allowed to start to converge again. This sequencing was repeated 10 times, without any user interference. The simulation was then continued until an approximate steady-state solution was reached. In each of the simulations, the MHD code was run to a steady state using local time stepping. This technique was shown by Ridley et al. (2002) to be a very good approximation for the real magnetosphere during time periods in which the IMF was not varying significantly. Since these simulations are purely hypothetical events, we neglect the time dependence of the 
magnetosphere, and simply focus on the nearly steady state solutions.

Each simulation was run with a fixed density of $28 \mathrm{~cm}^{-3}$ at the inner boundary, while the temperature (or pressure) and magnetic field was allowed to adjust to the given solution. The velocity was specified by the ionospheric boundary condition, as described in Ridley et al. (2004), where the ionospheric Pedersen conductance was fixed at a value of 4 mhos. While using a constant conductance in the ionosphere is unphysical, the auroral conductance is driven nonlinearly by the MHD solution. Therefore, the dependence of the ionospheric cross polar cap potential on the upstream conditions would be difficult to determine if the conductance were allowed to change as the field-aligned currents changed. In addition, a 4 mhos conductance represents a reasonable global average. Further studies will describe the relationship between the auroral conductance and the solar wind electric field, as the Mach number is lowered. A zero-gradient boundary condition was used on the side and back boundaries of the simulation, which allowed structures encountering them (such as Alfvén wings) to not reflect too badly. Some difficulties with dealing with sub-Alfvénic flows near boundaries will be discussed below.

\section{Results}

Figure 2 shows a series of simulation results of the magnetospheric electric and magnetic field as the IMF $B_{z}$ component changes from $-5 \mathrm{nT}$ to $-60 \mathrm{nT}$. For each of the runs, the solar wind density is $5 \mathrm{~cm}^{-3}$, the flow velocity is $400 \mathrm{~km} / \mathrm{s}$, and the temperature is $250000 \mathrm{~K}$. The Alfvénic Mach number, therefore, changes from 8.2 to 0.7 . The Y-component of the electric field is shown as a color contour, while the magnetic field lines in the $Y=0$ plane are traced. The spacing between the magnetic field lines do not necessarily indicate the strength of the field. The same starting points for the field-line tracing is used in each figure, though.

The top row represents the magnetosphere during nominal to disturbed conditions (i.e. $B_{z}=-5$ and $-15 \mathrm{nT}$, respectively), the second row reflects more disturbed periods (i.e. $B_{z}=-25$ and $-35 \mathrm{nT}$ ), while the bottom row shows extreme IMF conditions (i.e. $B_{z}=-45$ and $-60 \mathrm{nT}$ ). During nominal conditions, the magnetosphere appears quite "normal", where there is a well formed tail that extents far downstream of the Earth. At $B_{z}=-15 \mathrm{nT}$, the "tail" is still quite long - the electric field is reduced from the solar wind conditions and the magnetic field has a strong kink near the Equator to approximately $100 R_{E}$ downstream of the Earth. Beyond this distance, the electric field and magnetic field appear to be much more solar wind like.

As the Mach number decreases more, the "tail" becomes shorter, and the Alfvén wings structure is more prevalent. The magnetosphere no longer looks like the classic picture of a magnetosphere. A region of low electric field extends from the closed field region up (and down, which is not shown) at $32^{\circ}$ (for the $M_{a}=1.6$ case) to infinity. While it can not go all the way to infinity, it would extend out into the solar wind and IMF until the conditions change significantly. In planetary systems, such as Jupiter and Saturn, Alfvén wings extend from the moon (i.e., Io, Ganymede, Titan, etc.) to the main planet's ionosphere. Many studies have examined how the currents that exist on the edge of the Alfvén wing interact with the planet's ionosphere (e.g. Crary and Bagenal, 1997). The reason that the Alfvén wings do not go to infinity in the simulations shown here, is that (1) the resolution is worse in the outer wing, so it diffuses away; and (2) the wing interacts with the boundary of the model, so the solution near the boundary becomes corrupt. With a large enough box, and enough cells, the Alfvén wings would extend further outwards. This can be shown by increasing the box size and the number of cells and seeing if the same features are visible. This is indeed the case, since our initial runs were with a box size only extending to $128 R_{E}$ in $Z$. All of the same features were observed in the initial simulations (not shown), but when the box was extended, the Alfvén wing grew to be the sizes observed in Fig. 2.

\subsection{Electric field within the Alfvén wing}

An interesting aspect of the Alfvén wing, that can be observed in Fig. 2, is that as the electric field in the solar wind grows larger, the electric field in the Alfvén wing does not appear to change dramatically. There is a moderate change in the electric field in the Alfvén wing as $B_{z}$ decreases from -5 to $-15 \mathrm{nT}$, and again from -15 to $-25 \mathrm{nT}$ (all of the color tables are the same), but the change from $B_{z}=-25$ to $-35 \mathrm{nT}$ is reduced.

Neubauer (1980) showed that the electric field in the Alfvén wing $\left(E_{A}\right)$ can be described as:

$E_{A}=\frac{2 \Sigma_{A}}{2 \Sigma_{A}+\Sigma_{P}} E_{s w}$,

where $E_{s w}$ is the solar wind electric field, $\Sigma_{P}$ is the conductivity of the object, $\Sigma_{A}$ is the conductivity of the Alfvén wing, which is given as:

$$
\Sigma_{A}=\frac{1}{\mu_{0} V_{A} \sqrt{1+M_{A}^{2}}},
$$

and $\mu_{0}$ is the permiability of free space. Equation (1) is derived through current generation and closure considerations as the Alfvén Mach number decreases, the magnetic field becomes more rigid and can not support as much current. Taken at its most extreme, with a Mach number of zero, there will be no current, because either there is no plasma to drive a current, or no velocity to cause distortions in the field.

Figure 3 shows how the conductivity in the Alfvén wing and the electric field within the Alfvén wing changes as a function of $B_{z}$. The vertical dotted line shows where the solar 

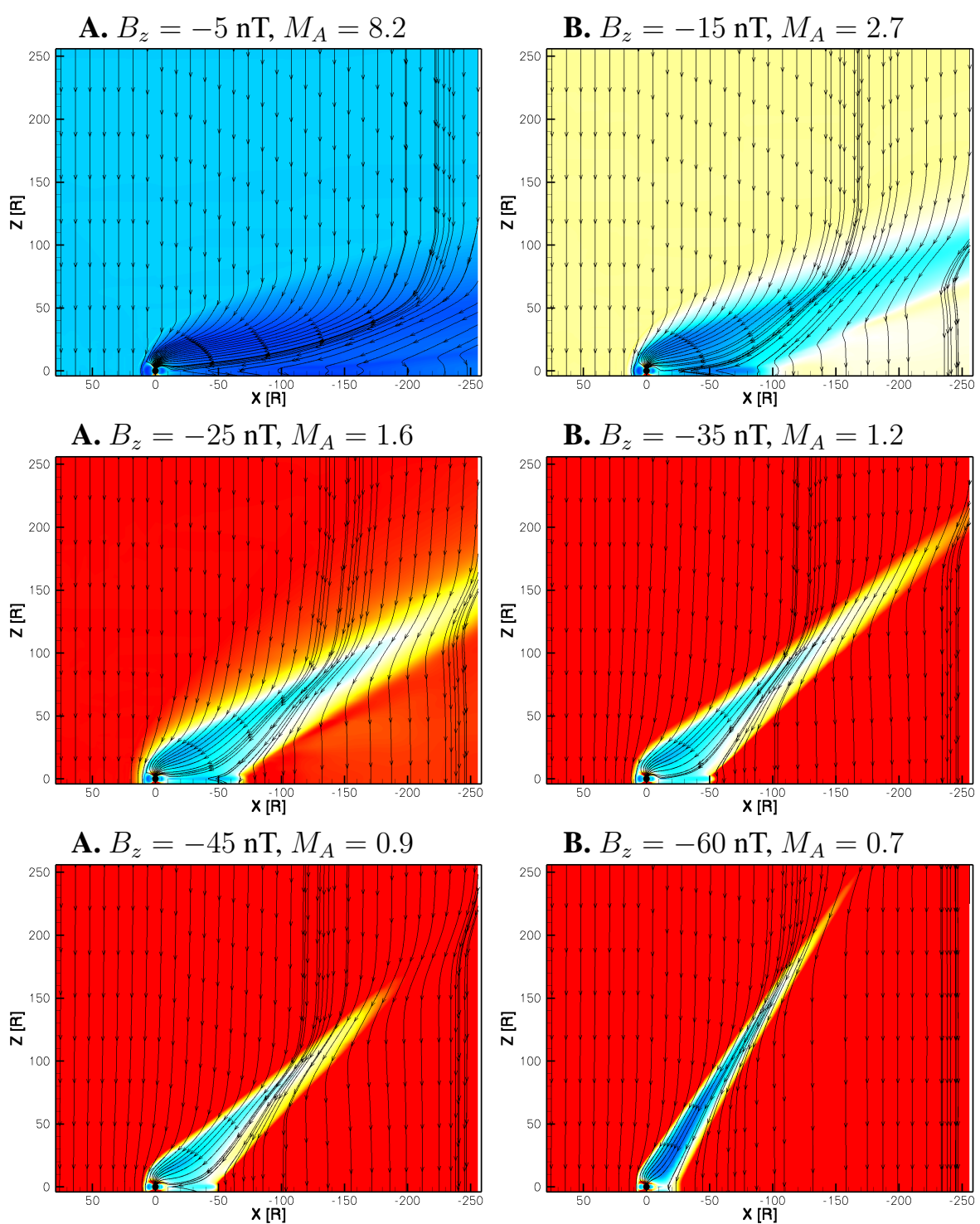

Fig. 2. The magnetospheric electric and magnetic fields in the $Y=0$ plane as a function of interplanetary magnetic field $B_{z}$. The electric field is shown as a color contour (dark blue is zero, while red is $10 \mathrm{mV} / \mathrm{m}$ ). Magnetic field lines are shown as as traces.

wind becomes sub-Alfvénic. The conductivity in the Alfvén wing becomes lower as the magnetic field becomes stronger, but the slope decreases. While the value asymptotes towards zero, it does this very slowly.

The electric field within the Alfvén wing increases with decreasing $B_{z}$, but at a decreasing rate. The dashed line in Fig. 3 shows the solar wind electric field, which linearly increases with decreasing $B_{z}$. The electric field (Eq. 1) can be rewritten as:

$$
E_{A}=\frac{2 V B}{2+\Sigma_{P} B \sqrt{\frac{\mu_{o}}{\rho}\left(1+M_{A}^{2}\right)}}
$$

As $B$ becomes large, the value asymptotes to:

$$
E_{A}=\frac{2 V \sqrt{\rho}}{\Sigma_{P} \sqrt{\mu_{o}}}
$$

since $M_{A}$ goes to zero as $B$ grows. For a solar wind velocity of $400 \mathrm{~km} / \mathrm{s}$ and a density of $5 \mathrm{~cm}^{-3}$, the asymptotic value is $0.016 \mathrm{~V} / \mathrm{m}$. This saturating of the electric field within the Alfvén wing has a consequence on the ionospheric cross polar cap potential.

\subsection{Ionospheric Cross Polar Cap Potential}

It has been shown in a number of studies that the ionospheric cross polar cap potential (CPCP) saturates during strong driving of the IMF and solar wind, such as during the 14 June 

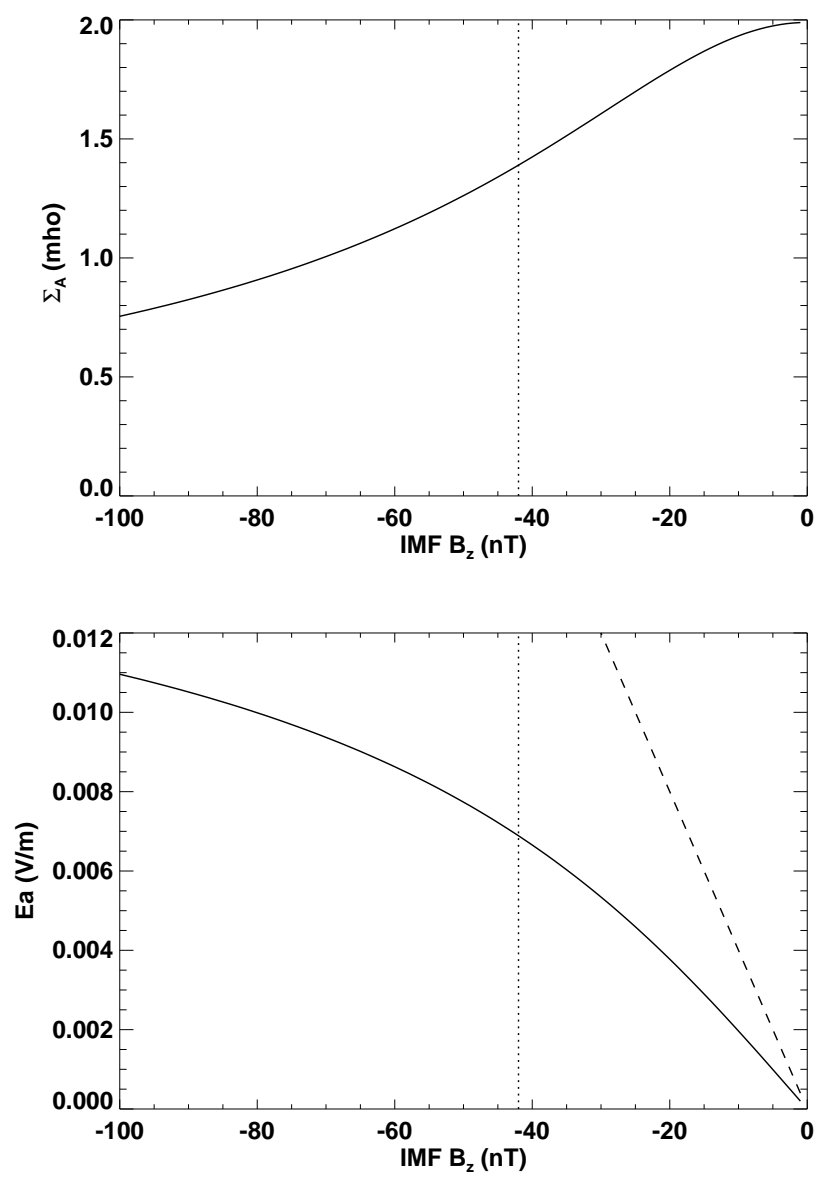

Fig. 3. $\Sigma_{A}$ (top) and $E_{A}$ (bottom).

2000 event. Only a few studies have attempted to describe why the potential saturates. The most widely discussed is by Siscoe et al. (2002), which describes the saturation of the CPCP as an internal mechanism, caused by the region1 currents reducing the strength of the magnetic field near the magnetopause. Many studies have shown that data support the findings of Siscoe et al. (2002), but with some differences in the ionospheric conductivity needed to produce saturation (e.g. Shepherd et al., 2002, 2003; Ober et al., 2003; Boudouris et al., 2004; Hairston et al., 2005). The ionospheric conductance is thought to strongly control the cross polar cap potential (Ridley et al., 2004), and therefore could possible cause it to saturate. This idea was examined by Merkine et al. (2003) and Nagatsuma (2004).

Other studies have described the saturation as possibly being caused by an external mechanism. The first such study was conducted by Reiff et al. (1981), who compared in-situ measurements of ionospheric plasma flows (or electric fields and the resulting potentials) to different magnetospheric coupling functions (e.g. Kan and Lee, 1979). They found that using an amplified magnetic field (due to the bow shock compression) worked best, but that the amplified field had to be limited to get the best fits. The best amplification factor was

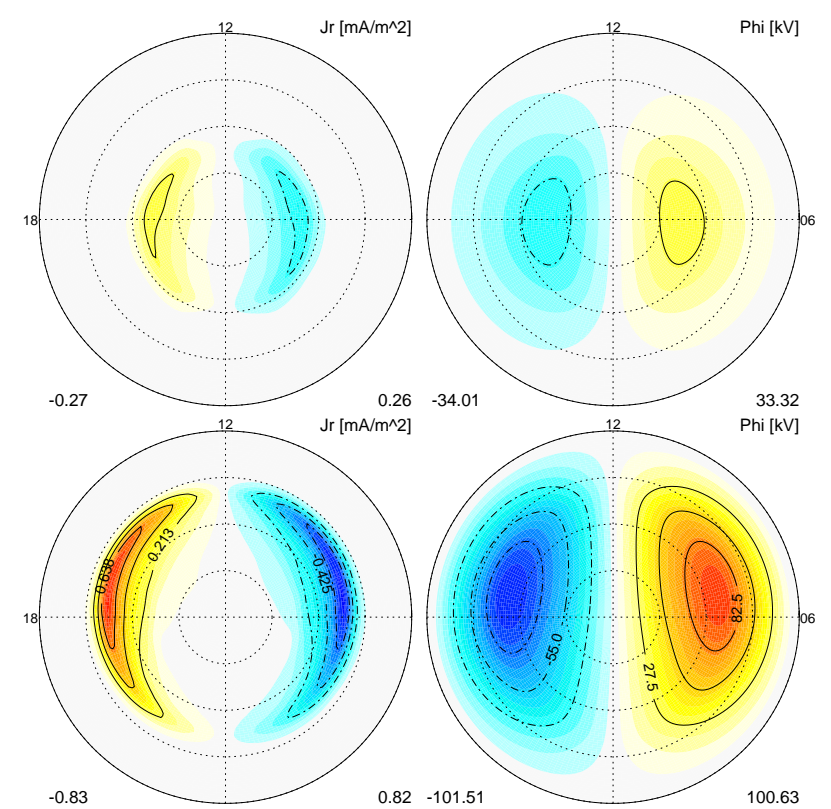

Fig. 4. Field-aligned current (left) in $\mu A / \mathrm{m}^{2}$ and potential (right) in $\mathrm{kV}$ for the nominal (top) and sub-Alfvénic (bottom) cases. The North pole is the center, $50^{\circ}$ is the outer ring, noon is at top, and dawn is to the right.

$7-8$, with a limiting value of $\sim 60$ (corresponding to a maximum IMF of $\sim 8 \mathrm{nT}$ ).

Recently, Ridley (2005) showed that the saturation of the ionospheric CPCP was correlated with time periods in which the solar wind Alfvén Mach number was reduced below approximately four. They showed that the potential could be modeled quite well by including the Mach number in the empirical relationship. The physical explanation offered was that through the bow shock, the magnetic field is compressed by a factor of four during a strong shock, but when the Mach number is reduced, the compression factor goes down - reaching one (i.e. no compression) when the Mach number is one. This is similar to the Reiff et al. (1981) study, but putting a relationship into the empirical formula instead of limiting value.

Figure 4 shows the ionospheric field-aligned currents (FACs) and the potential for the two cases: for $B_{z}=-5 \mathrm{nT}$ and $B_{z}=-100 \mathrm{nT}$. The structures within these plots do not significantly differ, except for the FACs being stronger and at lower latitude for the $B_{z}=-100 \mathrm{nT}$ case, resulting in a stronger and lower latitude potential. There is no real difference in the shapes of the patterns, implying that the coupling between the solar wind and magnetosphere may be a continuum instead of a fundamentally different processes controlling the magnetosphere during sub- and super-Alfvénic solar wind conditions.

Figure 5 shows the modeled ionospheric cross polar cap potential as a function of IMF $B_{z}$ as the $B_{z}$ is reduced from $-5 \mathrm{nT}$ to $-100 \mathrm{nT}$ for a solar wind density of $5 \mathrm{~cm}^{-3}$, a flow 


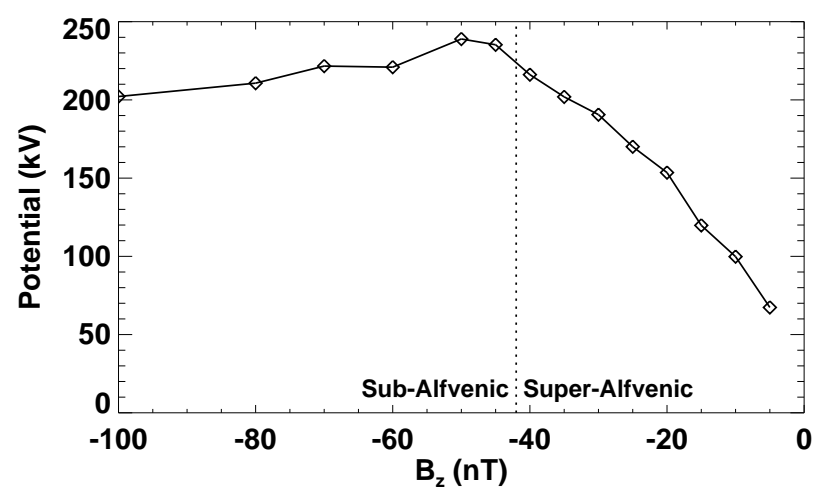

Fig. 5. A plot of the ionospheric cross polar cap potential as a function of IMF $B_{z}$. The diamonds represent values at which the MHD simulations were conducted.

velocity of $400 \mathrm{~km} / \mathrm{s}$, and a temperature of $250000 \mathrm{~K}$. This figure shows that when $B_{z}$ is small, the potential rises quickly with the increasingly southward IMF. As $B_{z}$ becomes large, though, the increase in the potential is smaller, and eventually, there is no increase in potential. This is commonly called the saturation of the cross polar cap potential, and can be approximated fairly well with an exponential fit. A vertical line differentiates sub- and super-Alfvénic solar wind conditions. It is clear that the transition to saturation occurs close to this point.

The ionospheric potential is an integral of the electric field of the Alfvén wing across the effective width of the reconnection site $\left(e_{r} \pi R_{m s}\right)$ similar to what was done in Siscoe et al. (2002):

$\Psi_{p c}=e_{r} \pi R_{m s} E_{a}+\Psi_{v}$,

where $e_{r}$ is the fraction of the dayside magnetosphere undergoing reconnection, which we take to be 0.1 (this may change, but variations in $e_{r}$ are not considered here), $\Psi_{v}$ is a viscous interaction potential, and is typically taken to be about 30 kV (e.g. Papitashvili et al., 1994; Burke et al., 1999), although this can vary, and may be dependent upon the solar wind density and flow velocity (e.g., Boyle et al., 1997). $R_{m s}$ is the radius of the magnetosphere given by a pressure balance between the solar wind and magnetospheric pressures:

$R_{m s}=\left(\frac{\left(2 B_{E}\right)^{2}}{2 \mu_{0} P_{s w}}\right)^{\frac{1}{6}} R_{E}$.

$B_{E}$ is the equatorial magnetic field value, $R_{E}$ is the radius of the Earth, and $P_{s w}$ is the solar wind pressure, which can include both the ram pressure and the magnetic pressure, since the magnetic field is a significant fraction of the total solar wind pressure at these strong field strengths.

Figure 6 shows the ionospheric potential as a function of $B_{z}$, as specified by Eq. (5) (solid line). The dashed line shows the integral of the solar wind electric field across the same reconnection line (with the addition of the viscous interaction

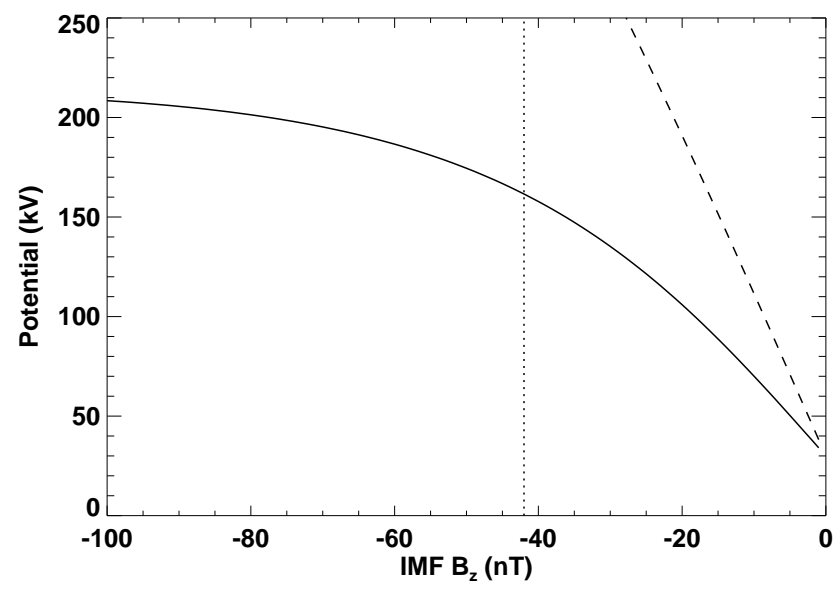

Fig. 6. The ionospheric cross polar cap potential as a function of $B_{z}$, as specified by Eq. (5).

potential, as specified in Eq. 5). The ionospheric potential is shown to saturate at a value just over $200 \mathrm{kV}$, which is a similar value as has been specified by other studies (Nagatsuma, 2002; Hairston et al., 2005). Interestingly, the reason that the potential saturates at a much lower $B_{z}$ than the electric field (i.e. Fig. 3), is that the size of the magnetosphere decreases as the magnetic field becomes larger. When the magnetic field pressure in the solar wind is included in Eq. (6), the length of the reconnection line decreases in size as the field becomes larger. This was discussed in Ridley (2005). The slow saturating of the electric field, in addition to the slow decrease in size of the reconnection line, causes the potential to saturate faster than one would expect just by examining the Alfvén wing electric field.

The MHD predicted saturation potential (as shown in Fig. 5) matches the predicted saturation potential (Fig. 6) quite well. The MHD potentials do tend to rise faster and level out faster with respect to $B_{z}$ than the predicted potential, but overall, the comparison is quite good.

If other components of the IMF change, a more accurate specification of the polar cap potential would be:

$\Psi_{p c}=V B_{y z} \sin ^{2}(\theta / 2) \frac{2 \Sigma_{A}}{2 \Sigma_{A}+\Sigma_{P}} e_{r} \pi R_{m s}+\Psi_{v}$,

where $B_{y z}=\sqrt{B_{z}^{2}+B_{y}^{2}}$, and $\theta=\cos ^{-1}\left(B_{z} / B_{y z}\right)$, and $V$ is the solar wind speed. The first term contains the reconnection electric field term $\left(V B_{y z} \sin ^{2}(\theta / 2)\right)$, given by Sonnerup (1974) and Kan and Lee (1979), the Alfvén wing shielding term, specified in Eq. (1), and the length of the reconnection line, as described above. This equation is identical to Eq. (5) for purely southward IMF (i.e. $B_{y}=0$ and $\theta=180^{\circ}$ ), and is similar to the Siscoe et al. (2002) formulation, in that it includes the efficiency of the reconnection rate, the coupling function based on $\theta$, the size of the magnetosphere, and an inverse relationship with the Pedersen conductance. 

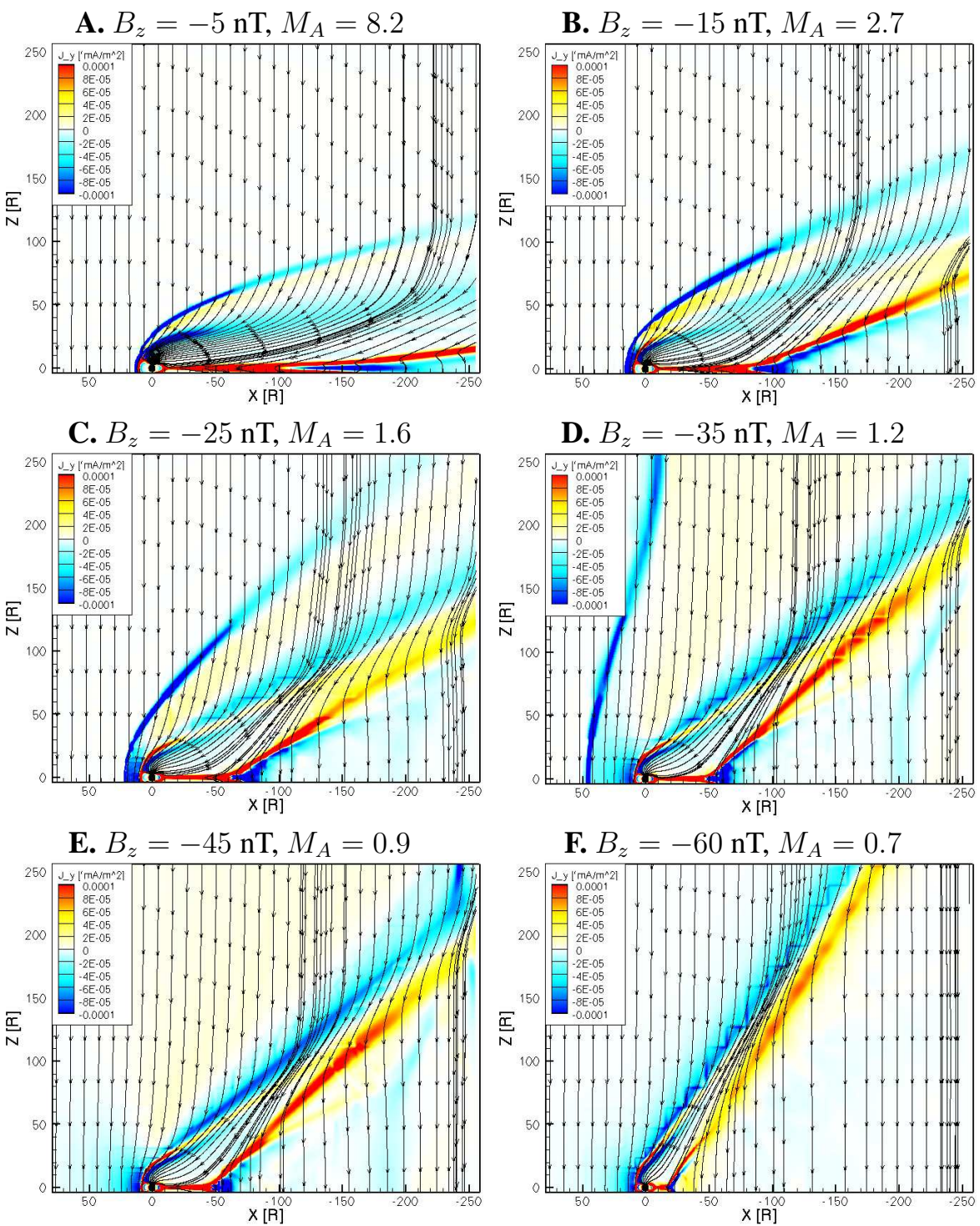

Fig. 7. The y-component of the magnetospheric current $\left(J_{y}\right)$ and the magnetic field traces in the $Y=0$ plane as a function of interplanetary magnetic field $B_{z} . J_{y}$ is shown as color contours. Magnetic field lines are shown as as traces.

\subsection{Magnetospheric currents}

Figure 7 shows the y-component magnetospheric currents $\left(J_{y}\right)$ as $B_{z}$ changes from -5 to $-60 \mathrm{nT}$, with the magnetic field lines traced as in Fig. 2. Examining the third row (E and F), the Alfvén wing is the dominant feature of the magnetosphere. There is a dusk-to-dawn current on the sunward side of the wing, and a dawn-to-dusk current on the back-side of the wing. Above and below the Alfvén wing, the field-lines are almost vertical, implying that they are very similar to the solar wind magnetic field. In the Alfvén wing, the field lines are almost straight, but at an angle to the IMF, with the kink in the field lines coincident with the currents.

Figures $7 \mathrm{c}$ and d show very similar features, although the current at the bow shock becomes a dominant current. Mag- netic field-lines can be traced from the solar wind down into the magnetospheric system in Fig. 7c. These field lines go through: (1) the bow shock current, which tilts them sunward; (2) a current that is associated with the shocked plasma accelerating back up to the solar wind speed, tilting the fieldlines antisunward (this current is light yellow); (3) the front current of the Alfvén wing that tilts the field-lines sunward; (4) in the far tail, the field lines next encounter the current associated with the back-end of the Alfvén wing and are bend back to almost vertical, although they are tilted slightly antisunward; and (5) field-lines that pass through the equatorial region between -75 and about $-150 R_{E}$ become distorted due to the fast flows out of the reconnection site in the tail, causing an over-expansion of the field-lines, therefore there is a current that brings them back to vertical in the equatorial 

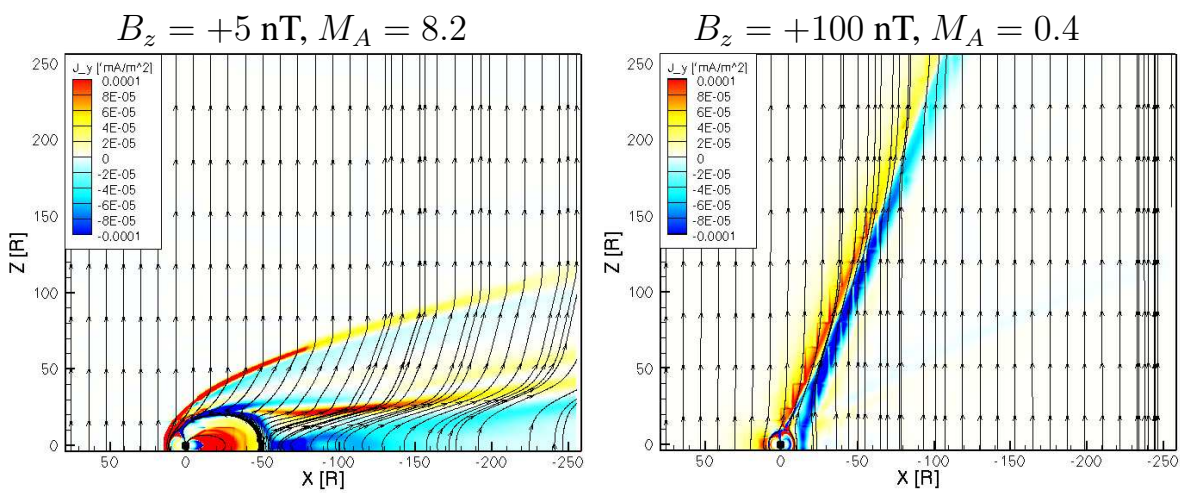

Fig. 8. The y-component of the magnetospheric current $\left(J_{y}\right)$ and the magnetic field traces in the $Y=0$ plane for $B_{z}=+5$ (left) and +100 (right) nT. $J_{y}$ is shown as color contours. Magnetic field lines are shown as as traces.

plane. Each of these currents can be observed in (a) and (b), although they may not be as obvious as in (c) and (d). In addition to these currents, there are many more current structures on the dayside magnetopause in the near-Earth region that will be discussed in detail in a future study.

From Figs. 2 and 7, the argument can be made that the magnetosphere acts as an Alfvén wing at all solar wind conditions. The largest difference between high and low Mach number conditions is the angle of the wing, and the location of the near-Earth currents. The wing structure can be most easily observed at high Mach numbers (i.e. low magnetic field values) in the tail current. This current bifurcates far downtail $\left(-100 R_{E}\right.$ for $B_{z}=-5 \mathrm{nT}$, and $-75 R_{E}$ for $B_{z}=-15 \mathrm{nT}$ ), and forms the back end of the Alfvern wing. The current on the sunward side of the magnetosphere is much weaker in the far-tail, but is quite strong in the lobe region for high Mach numbers.

\section{Northward IMF}

Alfvén wings should not be dependent upon sign of the IMF $B_{z}$. Figure 8 shows two simulations of the magnetosphere under northward IMF, one with a nominal Mach number (left) and one with a sub-Alfvénic Mach number (right). The Alfvén wing in the right plot is quite evident, and is very similar in appearance to the Alfvén wing simulations under southward IMF (e.g. Fig. 7f). In the left plot (i.e. nominal Mach number), the Alfvén wing can still be observed, as the two currents exiting the plot on the right side near $Z=30 R_{E}$, associated with the sunward (dawn-to-dusk current) and vertical (dusk-to-dawn current) tilting of the magnetic field. Interestingly, the magnetosphere is totally closed for these two simulations. Therefore the ionospheric conductance should have no influence on the electric field within the Alfvén wing, since currents have no field-line pathway from the Alfvén wing to the ionosphere. This aspect of the Alfvén wing will be examined in further studies.

\section{Discussion and conclusions}

The Earth's magnetosphere is embedded in the solar wind, which is almost exclusively a low magnetic field, high ram pressure environment. The solar wind Mach number, therefore, is almost always above four, implying that the Earth almost always has a strong bow shock a few $R_{E}$ upstream of the magnetopause. During time periods in which the interplanetary magnetic field increases and the solar wind density decreases, as is the case in magnetic clouds, the Alfvén Mach number can decrease to very small values though. Ridley (2005) showed that the Mach number can be less than 2-3 in many large magnetic storms, and even occasionally reduced below one.

In order to study this type of time period, it is beneficial to examine other bodies that have this type of external driving condition the majority of the time. For example, both Io and Ganymede exist in Jupiter's low density, high field strength magnetosphere. Both modeling and measurements from flybys of these objects show that they exhibit Alfvén wing structures (Neubauer, 1980; Kivelson et al., 1997; Linker et al., 1998; Ip and Kopp, 2002; Kopp and Ip, 2002), which are the direct result of the interaction between the external plasma and the body (i.e. the ionosphere or magnetosphere or the moon).

The University of Michigan MHD simulations of the Earth's magnetosphere show the formation of Alfvén wings also. Simulations are shown in which the solar wind Alfvén Mach number is reduced from a typical value (e.g. eight) to a value less than one. Alfvén wings are formed due to Alfvén waves propagating up the field-lines from the dayside magnetopause communicating to the solar wind that there is an object in the way. The angle of the wing is specified by $\theta=\operatorname{atan}\left(M_{A}^{-1}\right)$, or the angle between the solar wind velocity and the solar wind Alfvén velocity.

Modeling the Earth's magnetosphere during these types of events is nontrivial. Most models of the magnetosphere assume a super-Alfvénic solar wind, to ensure that waves can 
not propagate upstream and bounce off the boundary. In addition, as the Mach number is lowered towards one, the bow shock moves sunward. The Mach number therefore has to be above a certain value in order for the bow shock to not encounter the outer boundary. This can be compensated for in three ways: (1) move the upstream boundary of the simulation a long way away from the Earth, so lower Mach numbers can be simulated; (2) move the boundary away from the Earth and run for short periods of time, where the Mach number never exceeds 1 - there will be no bow shock to encounter the boundary and waves that are generated at the body won't be able to propagate all the way to the boundary and back before the simulation is completed; (3) modify the boundary conditions such that they absorb backwards propagating waves - this can be done by allowing one variable to float for each type of wave that may reach the boundary. We have simulated low Mach number flows (both for Earth and Io) using the University of Michigan MHD code using all three methods, and find that all of them work equally well.

Furthermore, the Alfvén wings are in a region of the simulation (i.e. above the poles) in which there is typically less resolution in the codes. One of the main challenges of global models is to resolve the dayside magnetopause and tail current sheet, therefore most of the resolution is concentrated near the equatorial plane. The Alfvén wings can typically be severely under resolved, causing them to be quite diffuse. For the simulations conducted above, $\sim 10000000$ cells were used, where typically $\sim 1000000$ cells are used for nominal magnetospheric simulations.

One of the characteristics of an Alfvén wing is that the electric field within the wing is reduced from the electric field within the surrounding plasma. This reduction in the electric field in the Alfvén wing may be the cause of the saturation of the ionospheric cross polar cap potential, as predicted by various data studies (e.g. Nagatsuma, 2002; Shepherd et al., 2002, 2003; Hairston et al., 2005). Our simulations show that the saturation occurs almost at the same point in which the solar wind becomes sub-Alfvénic.

Studies by Lopez et al. (2004) and Kataoka et al. (2005) described the control of the solar wind density on the ionospheric potential. They point out that as the magnetic field becomes larger, the density can have a larger influence on the potential. Both studies showed that this is most likely due to the solar wind Mach number. Most recently, Borovsky and Denton (2006) showed that the Siscoe et al. (2002) formulation can be rewritten in terms of the Alfvén Mach number and the Pedersen conductance, and that the saturation of the potential was most likely due to the decreasing Mach number.

All of the simulation results presented here are performed under the assumption of steady-state. This assumption is valid for slowly varying solar wind and IMF conditions, which may exist during magnetic clouds, which have slowly rotating magnetic fields. A crude estimation of how long it would take to set up the Alfvén wing can be estimated, since it is the controlled by Alfvén waves propagating up the magnetic field-lines. If we assume that the Alfvén wave speed in the solar wind is on the order of $200 \mathrm{~km} / \mathrm{s}$ (under strong driving), and the length scale of the Alfvén wing near the Earth is around $100 R_{E}$, the time-scale for the Alfvén wave is about one hour. This means that if the solar wind parameters are varying on a time scale of more than an hour, then it is probable that the Alfvén wings would appear.

This study predicts that Alfvén wings form during time periods in which the solar wind Mach number is reduced. It further suggests that the saturation of the CPCP is tied to the formation of Alfvén wings. Ridley (2005) showed that during 13 time periods in which there were strong solar wind and IMF driving conditions, both the Alfvén Mach number decreased to low values and that the ionospheric potential showed clear indications of saturation. This implies that both data and modeling results show that the saturation of the ionospheric cross polar cap potential is most likely caused by the formation of Alfvén wings above and below the Earth, and not by an internal mechanism. The most obvious way of further validating this idea is to examine magnetospheric satellite data during highly disturbed time periods to determine whether Alfvén wings form and what the electric field is in the Alfvén wing. The time-scales of the Alfvén wing formation can be tested by modeling some of the timeperiods discussed in Ridley (2005).

Acknowledgements. A. J. Ridley greatly appreciates the many conversations on Alfvén wings with K. C. Hansen and M. Kivelson. This research was supposed by NSF through grants ATM-0077555, ATM-0417839, and ATM-0325332, the DoD MURI program grant F4960-01-1-0359, and NASA grant NNG04GK18G.

Topical Editor I. A. Daglis thanks W. H. Ip and J. Borovsky for their help in evaluating this paper.

\section{References}

Borovsky, J. E. and Denton, M. H.: Differences between CMEdriven storms and CIR-driven storms, J. Geophys. Res., 111, A07S08, doi:10.1029/2005JA011447, 2006.

Boudouris, A., Zesta, E., Lyons, L., and Anderson, P.: Evaluation of the Hill-Siscoe transpolar potential saturation model during a solar wind dynamic pressure pulse, Geophys. Res. Lett., 31, L23 802, doi:10.1029/2004GL021252, 2004.

Boyle, C., Reiff, P., and Hairston, M.: Empirical polar cap potentials, J. Geophys. Res., 102, 111-125, 1997.

Burke, W., Weimer, D. R., and Maynard, N. C.: Geoeffective interplanetary scale sizes derived from regression analysis of polar cap potentials,, J. Geophys. Res., 104, 9989-9994, 1999.

Crary, F. and Bagenal, F.: Coupling the plasma interaction at Io to Jupiter, Geophys. Res. Lett., 24, 2135-2138, 1997.

Drell, S., Foley, H., and Ruderman, M.: Drag and propulsion of large satellites in the ionosphere: An Alfvén propulsion engine in space, J. Geophys. Res., 70, 3131, 1965.

Hairston, M., Drake, K. A., and Skoug, R.: Saturation of the ionospheric polar cap potential during the October-November 
2003 superstorms, J. Geophys. Res., 110(A9), A09526, doi:10.1029/2004JA010864, 2005.

Ip, W.-H. and Kopp, A.: Resistive MHD simulations of Ganymede's magnetosphere 2. Birkland currents and particle energetics, J. Geophys. Res., 107, 1491, doi:10.1029/2001JA005072, 2002.

Kan, J. and Lee, L.: Energy coupling function and solar wind magnetosphere dynamo, Geophys. Res. Lett., 6, 577-580, 1979.

Kataoka, R., Fairfield, D. H., Sibeck, D. G., Rastätter, L., Fok, M.-C., Nagatsuma, T., , and Ebihara, Y.: Magnetosheath variations during the storm main phase on 20 November 2003: Evidence for solar wind density control of energy transfer to the magnetosphere, Geophys. Res. Lett., 32, L21 108, doi:10.1029/2005GL024495, 2005.

Kivelson, M., Khurana, K., Coroniti, F., Joy, S., Russell, C., Walker, R., Warnecke, J., Bennett, L., and Polanskey, C.: Magnetic field and magnetosphere of Ganymede, Geophys. Res. Lett., 24, 2155-2158, 1997.

Kopp, A. and Ip, W.-H.: Resistive MHD simulations of Ganymede's magnetosphere 2. Time variabilities of the magnetic field topology, J. Geophys. Res., 107, 1490, doi:10.1029/2001JA005071, 2002.

Linker, J., Khurana, K., Kivelson, M., and Walker, R.: MHD Simulations of Io's interaction with the plasma torus, J. Geophys. Res., 103, 19867-19891, 1998.

Lopez, R., Wiltberger, M., Hernandez, S., and Lyon, J.: Solar wind density control of energy transfer to the magnetopause, Geophys. Res. Lett., 31(8), L08804, doi:10.1029/2003GL019253, 2004.

Merkine, V., Papadopoulos, K., Milikh, G., Sharma, A., Shao, X., Lyon, J., and Goodrich, C.: Effects of the solar wind electric field and ionospheric conductance on the cross polar cap potential: Results of global MHD modeling, Geophys. Res. Lett., 30, 2180, doi:10.1029/2003GL017903, 2003.

Nagatsuma, T.: Saturation of polar cap potential by intense solar wind electric fields, Geophys. Res. Lett., 29, 1422, doi:10.1029/2001GL014202, 2002.

Nagatsuma, T.: Conductivity dependence of cross-polar potential saturation, J. Geophys. Res., 109, A04210, doi:10.1029/2003JA010286, 2004.

Neubauer, F.: Nonlinear standing Alfvén wave current system at Io: Theory, J. Geophys. Res., 85, 1171-1178, 1980.
Ober, D., Maynard, N., and Burke, W.: Testing the Hill model of transpolar saturation, J. Geophys. Res., 108, 1467, doi:10.1029/2003JA010154, 2003.

Papitashvili, V., Belov, B., Faermark, D., Feldstein, Y., Golyshev, S., Gromova, L., and Levitin, A.: Electric potential patterns in the northern and southern polar regions parameterized by the interplanetary magnatic field, J. Geophys. Res., 99, 13 251-13 262, 1994.

Powell, K., Roe, P., Linde, T., Gombosi, T., and De Zeeuw, D. L.: A solution-adaptive upwind scheme for ideal magnetohydrodynamics, J. Comp. Phys., 154, 284-309, 1999.

Reiff, P., Spiro, R., and Hill, T.: Dependence of polar cap potential on interplanetary parameters, J. Geophys. Res., 86, 7639-7648, 1981.

Ridley, A.: A new formulation for the ionospheric cross polar cap potential including saturation effects, Ann. Geophys., 23, 35333547, 2005, http://www.ann-geophys.net/23/3533/2005/.

Ridley, A., Hansen, K., Tóth, G., De Zeeuw, D. L., Gombosi, T., and Powell, K.: University of Michigan MHD results of the GGCM Metrics challenge, J. Geophys. Res., 107(A10), 1290, doi:10.1029/2001JA000253, 2002.

Ridley, A., Gombosi, T., and De Zeeuw, D. L.: Ionospheric control of the magnetospheric configuration: Conductance, Ann. Geophys., 22, 567-584, 2004, http://www.ann-geophys.net/22/567/2004/.

Shepherd, S., Ruohoniemi, J., and Greenwald, R.: Cross polar cap potentials measured with Super Dual Auroral Radar Network during quasi-steady solar wind and interplanetary magnetic field conditions, J. Geophys. Res., 107, 1094, doi:10.1029/2001JA000152, 2002.

Shepherd, S., Ruohoniemi, J., and Greenwald, R.: Testing the Hill model of transpolar potential with Super Dual Auroral Radar Network observations, Geophys. Res. Lett., 30, 1002, doi:10.1029/2002GL015426, 2003.

Siscoe, G. L., Erickson, G., Sonnerup, B., Maynard, N., Schoendorf, J., Siebert, K., Weimer, D., White, W., and Wilson, G.: Hill model of transpolar potential saturation: Comparisons with MHD simulations, J. Geophys. Res., 107, 1321, doi:10.1029/2001JA009176, 2002.

Sonnerup, B.: Magnetopause reconnection rate, J. Geophys. Res., 79, 1546-1649, 1974. 